\title{
Antibiotic trends of Klebsiella pneumoniae and Acinetobacter baumannii resistance indicators in an intensive care unit of Southern Italy, 2008-2013
}

Antonella Agodi ${ }^{1 *}$, Martina Barchitta ${ }^{1}$, Annalisa Quattrocchi ${ }^{1}$, Andrea Maugeri $^{1}$, Eugenia Aldisio ${ }^{2}$, Anna Elisa Marchese ${ }^{2}$, Anna Rita Mattaliano ${ }^{2}$ and Athanassios Tsakris ${ }^{3}$

\begin{abstract}
Background: The overuse of antimicrobials is one of the main factors responsible for the development and spread of antimicrobial resistance, together with other causes, such as intra- and inter-hospital spread of resistant microorganisms and infection control policies and practices. The objective of the present study is to report the trends of Klebsiella pneumoniae and Acinetobacter baumannii antimicrobial resistance indicators in an Italian intensive care unit (ICU) during a six-year period, from 2008 to 2013.
\end{abstract}

Methods: Susceptibility data and annual antibiotic consumptions in the ICU were retrospectively obtained from the clinical laboratory and the pharmacy. Trends over time of resistance rates (RRs) and of incidence densities of resistant isolates were determined by linear regression.

Results: Isolation density of A. baumannii increased significantly from 2008 (20.4 per 1,000 patient-days) to 2013 (58.1 per 1,000 patient-days) and of K. pneumoniae from 2010 (22.3 per 1,000 patient-days) to 2013 (55.9 per 1,000 patient-days). RRs of third-generation cephalosporins (3GCs)-resistant $K$. pneumoniae (from 2010: $41.9 \%$, to 2012: $87.0 \%$ ), of carbapenem-resistant K. pneumoniae (from 2008: $0 \%$, to 2013: $59.2 \%$ ), and of carbapenem-resistant A. baumannii (from 2008: $87.5 \%$, to 2013: $96.6 \%$ ) showed significant increasing trends. Carbapenems was the main antibiotic class consumed ( $24.9 \%$ of the total antimicrobial usage density), followed by $3 G C s$ ( $21.0 \%)$, fluoroquinolones (20.6\%), aminoglycosides (17.3\%), penicillins (15.1\%) and glycopeptides (1.1\%). Carbapenems consumption decreased from 2008 to 2012 and then increased in 2013. Glycopeptides consumption decreased from 2008 to 2011 and then increased in 2013. Aminoglycosides consumption decreased from 2008 to 2010 and increased from 2012 to 2013. Finally, 3GC, penicillins and fluoroquinolones consumptions decreased from 2012 to 2013.

Conclusions: RRs of carbapenem-resistant A. baumannii and of carbapenem- and 3GC-resistant K. pneumoniae were higher than those for Europe. Our findings highlight the necessity to implement an integrated system for monitoring not only consumption of antibiotics and resistance profiles but also the clonality of alert microorganisms in the ICU for effective infection control.

Keywords: Antimicrobial resistance, Resistance rates, Antimicrobial usage density

\footnotetext{
*Correspondence: agodia@unict.it

'Department of Medical and Surgical Sciences and Advanced Technologies

"GF Ingrassia", University of Catania, Via S. Sofia 87, 95123 Catania, Italy

Full list of author information is available at the end of the article
} 


\section{Background}

Antimicrobial resistance (AMR) is a severe threat to public health in Europe and worldwide, leading to growing costs, treatment failure, and mortality [1, 2]. AMR results in reduced efficacy of drugs, and limits the available treatment options. The magnitude of the problem worldwide, the impact of AMR on health and on costs for the healthcare sector, together with the societal impact, are still unknown [2]. Thus, surveillance of AMR is considered an essential component of an effective response to this problem, and results produced constitute a fundamental source of information on the burden and trends of resistance [1]. Unfortunately, high resistance rates (RRs) to antimicrobial agents have been observed among bacterial pathogens that cause healthcare-associated and community-acquired infections worldwide and significant gaps in surveillance, lack of standards for methodology, data sharing and coordination have been highlighted [2]. Especially Gram-negative bacteria, including multidrug resistant Acinetobacter baumannii and Enterobacteriaceae producing Extended-spectrum beta-lactamases (ESBL) and carbapenemases, have been associated to severe healthcare-associated infections and their occurrence has increased in the last decades [1, 3]. In Europe, the most recent report of the European Antimicrobial Resistance Surveillance Network (EARS-Net) describes a general increase of AMR in the Gramnegative pathogens including Klebsiella pneumoniae and $A$. baumanni [1]. In Italy, the results of the Italian Nosocomial Infections Surveillance in Intensive Care Units (SPIN-UTI) network revealed that $K$. pneumoniae and $A$. baumanni are among the most commonly isolated microorganisms in intensive care unit (ICU)-acquired infections [4-6].

The overuse of antimicrobials is one of the main factors responsible for the development and spread of AMR. Therefore, European countries increasingly implement actions to control AMR in the community and the hospital setting through rational use of antimicrobials. Information on antimicrobial consumption in Europe is a prerequisite for antibiotic stewardship and can be an important source for healthcare professionals and policy makers in order to monitoring progress towards a prudent use of antibiotics [7]. However, other factors, such as intra- and inter-hospital spread of resistant microorganisms, community contribution to resistance, and infection control policies and practices, may also play a role in determining the burden of resistance in a hospital and should be considered [8]. We have previous reported intra- and inter-hospital spread of K. pneumoniae clones in two Sicilian ICUs [9] and the emergence of a carbapenem-resistant $K$. pneumoniae clone in one of those ICUs [10]. Furthermore, recently, we have described the dissemination, in the two ICUs of clonally related isolates of carbapenem-resistant $A$. baumannii with simultaneous resistance to colistin, hypothesizing that prior carbapenem and colistin consumptions may have acted as triggering factors [11].

All these findings support the need of strict adherence to control measures to prevent the dissemination of multidrug-resistant microorganism, together with the monitoring of antibiotic consumption at local level to promote the judicious use of antibiotics. The objective of the present study is to report the trends of $K$. pneumoniae and A. baumannii resistance indicators in an Italian ICU during a six-year period, from 2008 to 2013.

\section{Methods}

\section{Setting}

A retrospective study was conducted, during the period 2008-2013, at an 8-bed interdisciplinary ICU of the Azienda Ospedaliero - Universitaria "Policlinico - Vittorio Emanuele" in Catania, Italy. The total number of patient-days for each year was collected at the unit level.

\section{Microorganisms and antibiotic susceptibility tests}

Data on microorganisms isolated from ICU patients were retrospectively obtained from the clinical laboratory of the hospital. Identification and susceptibility testing, including ESBL test, were performed using the Phoenix System (Becton Dickinson, Sparks, MD, USA). A laboratory-based surveillance, without patient-based data collection, was performed and the susceptibility data were collected for K. pneumoniae and A. baumannii regardless of whether they were associated with healthcare-associated or community-acquired infection or colonization, or whether they were from clinical or surveillance cultures. Particularly, data on carbapenem and 3GC -resistant K. pneumoniae and on carbapenemresistant $A$. baumannii were included in the present study. Isolates were classified as susceptible, intermediate or resistant according to Clinical and Laboratory Standards Institute guidelines [12] and, from 2012, to European Committee on Antimicrobial Susceptibility Testing guidelines [13]. Copy strains, defined as an isolate of the same species showing the same susceptibility pattern throughout a period of 1 month in the same patient, no matter what the site of isolation, were excluded [14]. For each species, the isolation density was calculated as the number of isolates per 1,000 patientdays. Antibiotic RRs were calculated as the number of resistant or intermediate isolates divided by the total number of isolates of the same species tested against the corresponding antibiotic multiplied by 100 . Furthermore, the incidence density of resistant isolates was calculated as the number of resistant or intermediate isolates per 1,000 patient-days. 


\section{Antibiotic consumption}

Data on annual antibiotic consumption in the ICU, from 2008 to 2013, were obtained from the pharmacy of the hospital. Consumption - that is the antimicrobial usage density (AD) - was expressed as defined daily dose (DDD) and was normalized per 1,000 patient-days. The DDD are the standard adult daily dose of an antimicrobial agent for a 1-day treatment defined by the World Health Organization [15]. Particularly, data on consumption of third-generation cephalosporins (3GCs: ceftriaxone, cefotaxime and ceftazidime), penicillins (piperacillin, piperacillin/tazobactam, ampicillin, amoxicillin), glycopeptides (vancomicin), carbapenems (imipenem, meropenem, and ertapenem), aminoglycosides (amikacin, gentamicin, tobramicin and netalmicin), and fluoroquinolones (levofloxacin and ciprofloxacin) were collected. Notably, gentamicin and ertapenem were introduced into the ICU in 2009 and 2011, respectively.

\section{Statistical analyses}

Statistical analyses were performed using the SPSS 22.0 statistical package (SPSS Inc., Chicago, IL, USA). Trends over time of RRs and of incidence densities of resistant isolates were determined by linear regression with the yearly data. Furthermore, rate ratios were computed to compare ADs between different years. Pearson's correlation coefficient (cc) was used to determine the relationship between antibiotic consumption and antibiotic RRs and incidence densities of resistant isolates. A p value $<0.05$ was considered statistically significant.

\section{Results}

\section{Microorganisms and antibiotic resistance}

During the six-year period, among the 5,529 nonrepetitive microbial isolates recovered from clinical or surveillance samples, Gram-negatives were the most frequently isolated microorganisms (48.6\%), followed by fungi (29.6\%) and Gram-positives (21.8\%). Particularly, a total of $475 \mathrm{~K}$. pneumoniae $(8.6 \%)$ and of $463 \mathrm{~A}$. baumannii $(8.4 \%)$ were isolated. Isolation density of $A$. baumannii increased significantly from 2008 (20.4 per 1,000 patient-days) to 2013 (58.1 per 1,000 patientdays) and of K. pneumoniae from 2010 (22.3 per 1,000 patient-days) to 2013 (55.9 per 1,000 patient-days) (Table 1).

Table 1 shows the RRs during the study period. RRs of 3GC-resistant K. pneumoniae (from 2010: $41.9 \%$, to 2012: $87.0 \%$ ), of carbapenem-resistant $K$. pneumoniae (from 2008: $0 \%$, to 2013: 59.2 \%), and of carbapenem-resistant A. baumannii (from 2008: 87.5 \%, to 2013: $96.6 \%$ ) showed significant increasing trends. Frequencies and incidence densities (per 1,000 patient-days) of ESBL-producing $K$. pneumoniae among 3 GC-resistant $K$. pneumoniae were shown in Table 1.
Considering incidence density of resistant isolates (per 1,000 patient-days), significant increasing trends were reported for 3GC-resistant K. pneumoniae (from 2010 to 2013), carbapenem-resistant K. pneumoniae (from 2008 to 2013), and carbapenem-resistant $A$. baumannii (from 2008 to 2013) (Table 1).

\section{Antibiotic consumption}

Antibiotic consumption over the 6-year period was 7,362 DDD per 1,000 patient-days (AD). Overall carbapenems (24.9\% of the total AD) was the main antibiotic class consumed, followed by 3GCs $(21.0 \%)$, fluoroquinolones (20.6\%), aminoglycosides (17.3\%), penicillins (15.1\%) and glycopeptides (1.1\%). Figure 1 shows the heterogeneity of the antibiotic consumption (in $\mathrm{AD}$ ), by antibiotic classes, and the time-trend distribution in the ICU.

In general, the consumption of each antibiotic class varied with years. Carbapenems consumption decreased from 2008 to 2012 (rate ratio 0.61; $95 \%$ CI 0.54-0.69) and then increased, by about 2-fold, in 2013 (rate ratio 1.81; 95 \% CI 1.61-2.02). Glycopeptides consumption decreased from 2008 to 2011 (rate ratio 0.15; $95 \%$ CI 0.06-0.35) and then intensely increased in 2013 (rate ratio 10.85; $95 \%$ CI 4.73-30.63). Aminoglycosides consumption decreased from 2008 to 2010 (rate ratio 0.15; 95 \% CI 0.12-0.18) whereas it increased from 2012 to 2013 (rate ratio 5.34; $95 \%$ CI 4.27-6.76). Finally, 3GCs (rate ratio 0.51; $95 \%$ CI $0.45-0.58$ ), penicillins (rate ratio $0.44 ; 95 \%$ CI $0.37-0.52$ ) and fluoroquinolones (rate ratio 0.77; $95 \%$ CI $0.67-0.89$ ) consumptions decreased from 2012 to 2013.

Figure 2 reports trends of incidence density of resistant isolates (per 1,000 patient-days) and of the corresponding antibiotic consumption (in $\mathrm{AD}$ ) during the study period. No correlations between the usage of antimicrobial agents and resistance data were found.

\section{Discussion}

Italy is one of the European countries with increasing spread of antimicrobial-resistant microorganisms, often multidrug-resistant [1] and with high antibiotic consumption in the hospital setting $[1,7,16]$. Several factors, such as antimicrobial consumption, clonal spread of resistant microorganisms, resistance mechanisms that might differ by species, the human and environmental reservoir, and infection control strategies, including screening policies, may play a role in the prevalence of antimicrobial-resistant pathogens in the hospital setting $[8,14]$. This issue is of interest especially in Italian ICUs where the highest prevalence of patients on antibiotic treatment is observed [17] and outbreaks due to multidrug-resistant $K$. pneumoniae and A. baumannii are frequently reported [9-11, 18]. Comparison of our 
Table 1 Isolation density, resistance rates and incidence density of resistant isolates during the period 2008 - 2013

\begin{tabular}{|c|c|c|c|c|c|c|c|}
\hline & \multicolumn{6}{|l|}{ Year } & \multirow[b]{2}{*}{$p$-value* } \\
\hline & 2008 & 2009 & 2010 & 2011 & 2012 & 2013 & \\
\hline \multicolumn{8}{|l|}{ Isolation density } \\
\hline \multicolumn{8}{|l|}{ (per 1000 patient-days) } \\
\hline Klebsiella pneumoniae & 38.0 & 48.5 & 22.3 & 31.9 & 41.6 & 55.9 & $\begin{array}{l}0.497(\mathbf{0 . 0 0 5} \\
\text { from } 2010 \text { to 2013) }\end{array}$ \\
\hline Acinetobacter baumannii & 20.4 & 19.3 & 29.8 & 49.0 & 50.3 & 58.1 & 0.002 \\
\hline \multicolumn{8}{|l|}{ Resistance rates, \% } \\
\hline \multicolumn{8}{|l|}{ (number of tested isolates) } \\
\hline 3GC-resistant $K$. pneumoniae & $88.7(53)$ & $69.4(62)$ & $41.9(43)$ & $67.2(64)$ & $87.0(69)$ & $81.6(98)$ & $\begin{array}{l}0.806(\mathbf{0 . 0 4 5} \\
\text { from } 2010 \text { to 2012) }\end{array}$ \\
\hline \multirow{2}{*}{$\begin{array}{l}\text { ESBL-producing } \text { K. pneumoniae } \\
\text { among 3GC-resistant } K \text {. pneumoniae }\end{array}$} & 100 & 100 & 100 & 44.2 & 98.3 & 93.8 & \multirow[t]{2}{*}{0.674} \\
\hline & (39) & $(42)$ & $(18)$ & $(43)$ & (59) & $(80)$ & \\
\hline Carbapenem-resistant $K$. pneumoniae & $0(53)$ & $9.7(62)$ & $2.3(43)$ & $37.5(64)$ & $63.8(69)$ & $59.2(98)$ & 0.010 \\
\hline Carbapenem resistant $A$. baumannii & $87.5(14)$ & $95.0(19)$ & $96.2(51)$ & $98.9(92)$ & $93.2(68)$ & $96.6(85)$ & 0.021 \\
\hline \multicolumn{8}{|l|}{$\begin{array}{l}\text { Incidence density of resistant isolates } \\
\text { (per 1,000 patient-days) }\end{array}$} \\
\hline 3GC-resistant K. pneumoniae & 25.9 & 25.1 & 8.9 & 19.3 & 32.8 & 35.5 & $\begin{array}{l}0.367(\mathbf{0 . 0 2 7} \\
\text { from } 2010 \text { to 13) }\end{array}$ \\
\hline $\begin{array}{l}\text { ESBL-producing } K \text {. pneumoniae } \\
\text { among } 3 G C \text {-resistant } K \text {. pneumoniae }\end{array}$ & 21.5 & 24.6 & 8.9 & 8.5 & 31.7 & 33.2 & 0.436 \\
\hline Carbapenem-resistant $K$. pneumoniae & 0 & 3.5 & 0.5 & 10.8 & 24.1 & 25.7 & 0.009 \\
\hline Carbapenem-resistant A. baumannii & 7.7 & 11.1 & 25.3 & 41.3 & 37.2 & 37.7 & 0.014 \\
\hline
\end{tabular}

*Linear regression

Significant values are indicated in bold font

Abbreviation: 3GC third-generation cephalosporin, ESBL Extended-spectrum beta-lactamase

data with those of the EARS-Net should be undertaken with caution, since European results are based on invasive isolates from blood or cerebrospinal fluid, thus not representative of isolates from other sites [1]. Our study confirms that carbapenem resistance is endemic for $A$. baumannii isolates, with dramatically higher RRs (about $95 \%)$ than those in the other European countries [1]. Furthermore, incidence density of this multidrugresistant pathogen increased significantly from 2008 to 2013 in our ICU, and consumption of carbapenems, after a significant decrease, increased in the last year of the study. Recently, it has been reported that the increasing use of carbapenems was associated with the increasing incidence of healthcare-associated infection due to imipenem-resistant $A$. baumannii, suggesting that caution in antibiotic use would play an important role in managing high RRs [19]. The results of our study, although not confirmatory, may also suggest that increasing carbapenems consumption contributed to increasing rate of drug-resistant organisms. However, in order to further explain high RRs of pathogens associated with infections in the ICUs, it has been suggested [11, 20, 21] that high RRs are correlated with high rates of antibiotic use, but increased and/or high RRs may also be due to transmission between patients. In fact, in the same ICU, we have recently described the dissemination of clonally related multidrug-resistant $A$. baumannii isolates [11].

In Europe, the percentage of carbapenem-resistant $K$. pneumoniae is already high and increasing in some countries. The highest RRs were reported by Greece followed by Italy $(28.8 \%)$ with statistically significant increasing trends [1]. Since carbapenem- resistant $K$. pneumoniae isolates are frequently found to be carbapenemaseproducing, these results highlight and confirm that surveillance, active screening of patients at high-risk, notification of health authorities, implementation of infection control measures and the prudent use of antimicrobials are key elements to contain the spread of carbapenem-resistant $K$. pneumoniae isolates [1, 22, 23]. In Italy since 2013 the Ministry of Health established a national surveillance of carbapenemase-producing Enterobacteriaceae (particularly, K. pneumoniae and Escherichia coli) [24]. In our ICU the percentage of carbapenem-resistant $K$. pneumoniae was higher than that one documented in Europe with a significant increasing trend, although consumption of carbapenems decreased until 2012 and increased in 2013. As described for A. baumanni, dissemination of clonally related carbapenem-resistant $K$. pneumoniae isolates has been also previously documented in our unit, thus 


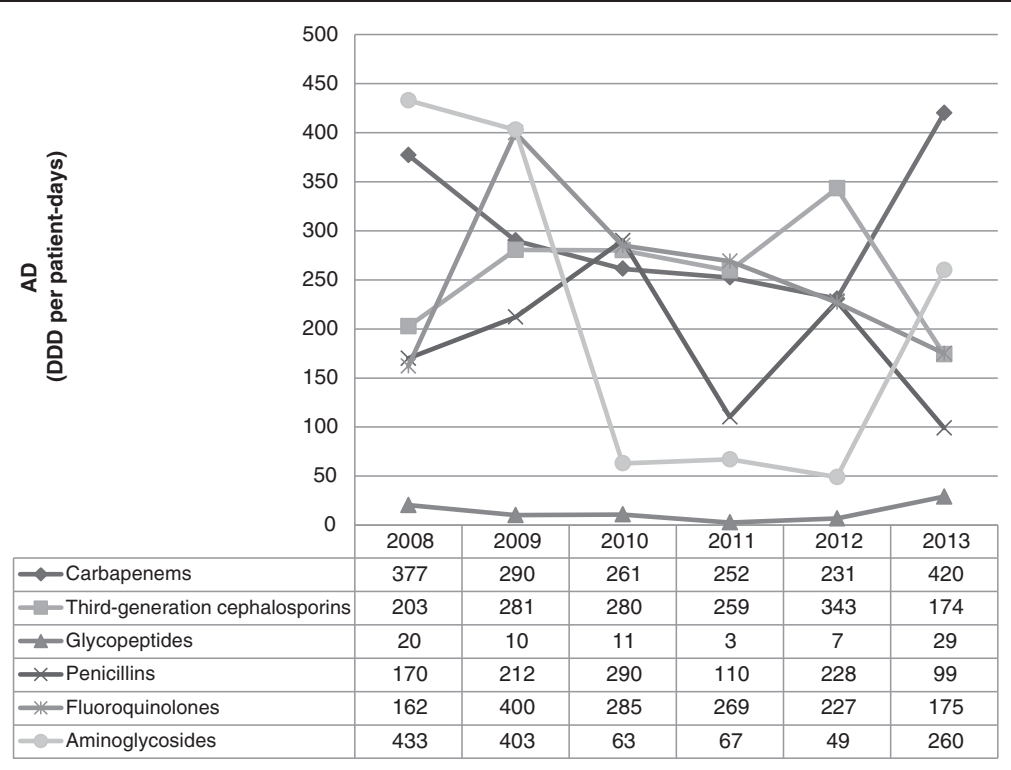

Fig. 1 Annual consumption of antibiotic classes from 2008 to 2013

contributing to the increasing trend of carbapenems resistance $[9,10]$. Notably, results on high carbapenem RRs of A. baumannii and K. pneumoniae are worrisome because carbapenems are last-line antibiotics. In addition, as previously reported, when carbapenems resistance is due to the presence of carbapenemases, accumulation of other resistance determinants, makes these isolates extensively drug- or pandrug-resistant, leaving few or no effective treatment options [22, 23, 25, 26].

In European countries, a large proportion of 3GCresistant $K$. pneumoniae isolates, ranging between $85 \%$ and $100 \%$, was ascertained as ESBL-positive [1]. In our study, frequencies of ESBL-producing $K$. pneumoniae among 3GC-resistant isolates were very high, reaching $100 \%$ in 2008, 2009 and 2010. Furthermore, the incidence density of ESBL producers increased, although not significantly, from 2010 to 2013. The high percentage of ESBL-producing $K$. pneumoniae complicates the treatment of serious infections caused by these bacteria leading to a significant increase in carbapenems use, considered the first line class for ESBL producer, with a consequent impact on the emergence of resistance to these antibiotics [1].

In our ICU the percentage of 3GC-resistant $K$. pneumoniae isolates was higher than that one reported for Italy $(47.7 \%)$ [1]. Furthermore, the incidence density of resistant isolates increased significantly from 2010 to 2012 together with the 3GC consumption. In 2013, the 3GC consumption decreased but the incidence density of resistant isolates increased with no evidence of clonal dissemination of 3GC-resistant $K$. pneumoniae in the ICU. Interestingly, in the German ICUs the dramatic increase of 3GC-resistant $K$. pneumoniae has been associated with an increased trend of antibiotic usage [14].

Finally, in the present study no correlations between resistance data and the corresponding antibiotic consumption were observed, thus the multifaceted nature of the spread and emergence of resistance can, at least partly, explain this result. In fact, although it is clear that antibiotics may act as promoters of resistance inducing key processes for the emergence and spread of resistance, as mutagenesis, recombination and/or horizontal gene transfer $[8,14,19,27]$, this occurrence is also influenced by other factors than by antibiotic consumption alone. Antibiotic-resistant bacteria, as well as resistance genes, can spread from person to person to the environment, and then back to humans thus, infection prevention and control activities to limit the spread of resistant bacteria are crucial [28].

Furthermore, although DDD measurements are useful for comparison and benchmarking, they may not fully correlate with subsequent antibiotic resistance development due to the intrinsic biases [29].

Our study has the limitation of the retrospective study design as well as the fact that is conducted in a single institution in Italy, thus the results cannot be representative of all ICUs of Southern Italy. Moreover, the ecological study design cannot prove a causative relationship between the two factors. Thus, a prospective multicentre patient-based study design is needed to confirm these findings. Furthermore, in our study design, data on AMR are laboratory-based, thus precluding a patient-based evaluation (i.e. date of admission to the ICU, clinical data confirming infection and other information) 


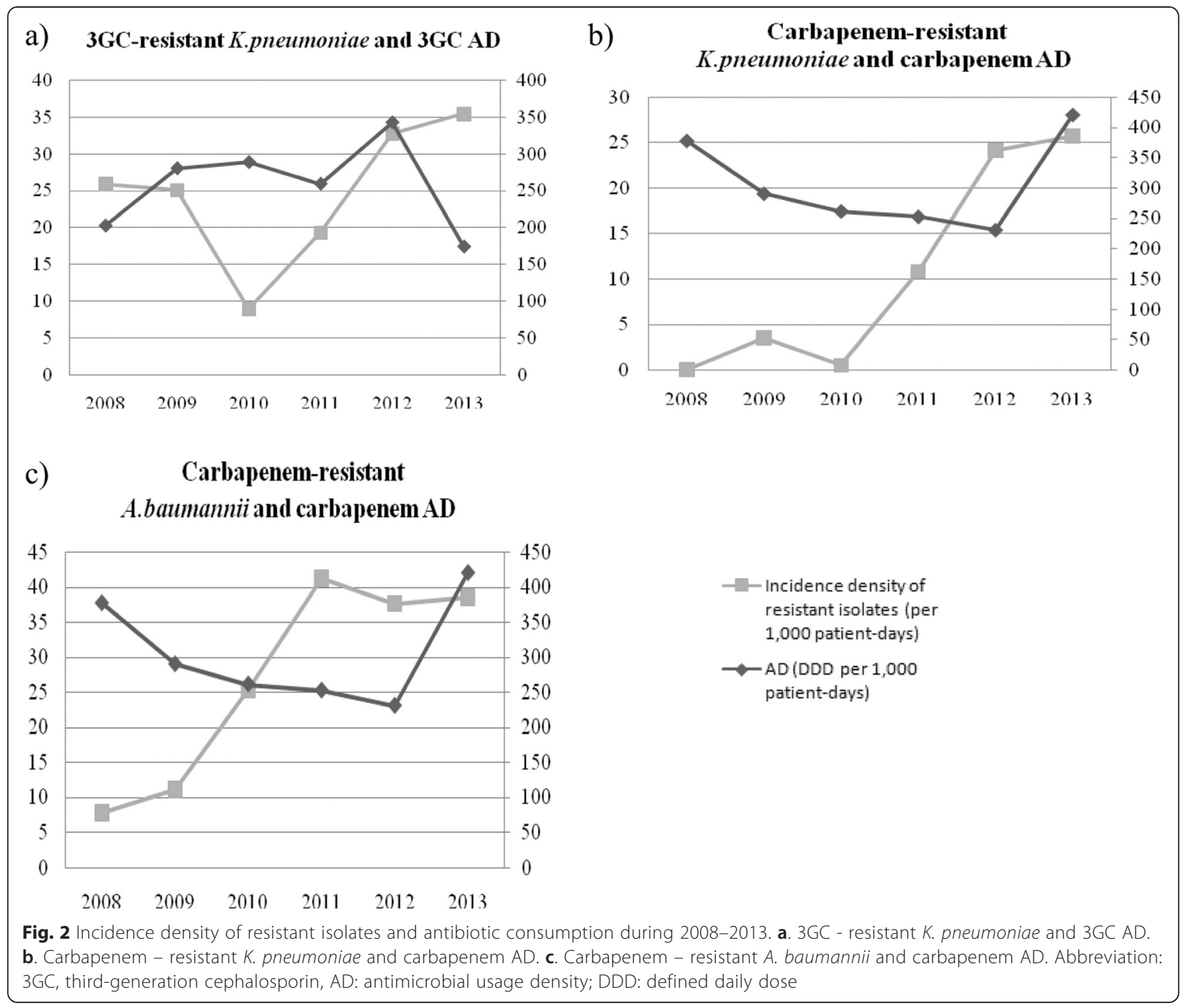

to differentiate whether microorganisms were associated with healthcare-associated or community-acquired infection or colonization. Since the emergence of drug-resistant microorganisms is commonly seen in nosocomial settings and invasive isolates could be of much more interest than microorganisms isolated from colonization episodes, microorganisms associated with infection episodes should be analyzed separately. Besides, no data about other factors such as patient's characteristics and the amount of antibiotics prescribed in the outpatient setting and potential confounders, such as antimicrobial stewardship interventions, were taken into account. Finally, outbreaks not investigated might have influenced resistance data.

\section{Conclusions}

The already high percentages and increasing trends of AMR K. pneumoniae and A. baumannii, described in this study, illustrate the continuous loss of effective antimicrobial therapy against these microorganisms and emphasize the need for comprehensive strategies targeting prudent use of antibiotics, since treatment options for infections with multiresistant bacteria are limited [1]. Besides, since very few effective antibiotics for multidrug-resistant Gram-negatives pathogens are likely to be launched in a close future, there is a critical need to implement strategies against the development of acquired resistance [30]. In our study, although increasing trends of AMR organisms and of antibiotic consumption were described, we did not confirm that increasing antibiotic consumption was correlated to increasing RRs, but we suggest that the introduction of phenotypic and molecular methods for enhanced surveillance and outbreak detection may result in improved infection control programs. Our findings also recommend the implementation of an integrated system for monitoring not only 
consumption of antibiotics and resistance profiles but also the clonality of alert microorganisms in the ICU for effective infection control.

\section{Abbreviations}

3GC: third-generation cephalosporins; AD: antimicrobial usage density; AMR: antimicrobial resistance; cc: correlation coefficient; DDD: defined daily dose; EARS-Net: european antimicrobial resistance surveillance network; ESBL: extended-spectrum beta-lactamases; ICU: intensive care unit; RRs: resistance rates.

\section{Competing interests}

The Authors declare that they have no competing interests

\section{Authors' contributions}

AA conceived the study design, coordinated the study, drafted, supervised and reviewed the manuscript; MB participated in study design, performed and interpreted the statistical analysis and drafted the manuscript; AQ performed and interpreted the statistical analysis and drafted the manuscript; AM performed and interpreted the statistical analysis and drafted the manuscript; EA collected data and interpreted the results; AEM: collected data and analyzed results in the laboratory; ARM participated in study design and reviewed the manuscript; AT drafted, supervised and reviewed the manuscript. All Authors read and approved the final manuscript.

\section{Acknowledgements}

We are grateful to Patrizia Bellocchi, Giacomo Castiglione, Rosario Cunsolo and Alida Imbriani for their collaboration in the research. We are grateful to Bench Srl, University of Catania for technical support.

\section{Author details}

${ }^{1}$ Department of Medical and Surgical Sciences and Advanced Technologies "GF Ingrassia", University of Catania, Via S. Sofia 87, 95123 Catania, Italy. ${ }^{2}$ Azienda Ospedaliero-Universitaria 'Policlinico-Vittorio Emanuele', Via S. Sofia, 78, 95123 Catania, Italy. ${ }^{3}$ Department of Microbiology, Medical School, University of Athens, 75 Mikras Asias Street, 11527 Athens, Greece. ${ }^{4}$ Department "GF Ingrassia", University of Catania, via S. Sofia, 87, 95123 Catania, Italy.

Received: 7 July 2015 Accepted: 23 October 2015 Published online: 03 November 2015

\section{References}

1. European Centre for Disease Prevention and Control. Antimicrobial resistance surveillance in Europe 2012. Annual report of the European Antimicrobial Resistance Surveillance Network (EARS-Net). Stockholm: ECDC; 2013.

2. World Health Organization. Antimicrobial resistance global report on surveillance 2014. http://www.who.int/drugresistance/documents/ surveillancereport/en/. 2014. Accessed 10 May 2015.

3. Tacconelli E, Cataldo MA, Dancer SJ, De Angelis G, Falcone M, Frank U, et al. ESCMID guidelines for the management of the infection control measures to reduce transmission of multidrug-resistant Gram-negative bacteria in hospitalized patients. Clin Microbiol Infect. 2014;20:1-55.

4. Agodi A, Auxilia F, Barchitta M, Brusaferro S, D'Alessandro D, Montagna MT, et al. Building a benchmark through active surveillance of ICU-acquired infections: the Italian network SPIN-UTI. J Hosp Infect. 2010;74:258-65.

5. Masia MD, Barchitta M, Liperi G, Cantù AP, Alliata E, Auxilia F, et al. Validation of intensive care unit-acquired infection surveillance in the Italian SPIN-UTI network. J Hosp Infect. 2010;76:139-42.

6. Agodi A, Auxilia F, Barchitta M, Brusaferro S, D'Alessandro D, Grillo OC, et al. Trends, risk factors and outcomes of healthcare-associated infections within the Italian network SPIN-UTI. J Hosp Infect. 2013;84:52-8.

7. European Centre for Disease Prevention and Control. Surveillance of antimicrobial consumption in Europe 2012. Stockholm: ECDC; 2014.

8. Goel N, Wattal C, Oberoi JK, Raveendran R, Datta S, Prasad KJ. Trend analysis of antimicrobial consumption and development of resistance in non-fermenters in a tertiary care hospital in Delhi, India. J Antimicrob Chemother. 2011;66:1625-30.

9. Agodi A, Barchitta M, Valenti G, Romeo MA, Giaquinta L, Santangelo C, et al. Cross-transmission of Klebsiella pneumoniae in two intensive care units: intra- and inter-hospital spread. J Hosp Infect. 2011;77:279-80.
10. Agodi A, Voulgari E, Barchitta M, Politi L, Koumaki V, Spanakis N, et al. Containment of an Outbreak of KPC-3 Carbapenemase-Producing Klebsiella pneumoniae in Italy. J Clin Microbiol. 2011;49:3986-9.

11. Agodi A, Voulgari E, Barchitta M, Quattrocchi A, Bellocchi P, Poulou A, et al. Spread of a carbapenem- and colistin-resistant Acinetobacter baumannii ST2 clonal strain causing outbreaks in two Sicilian hospitals. J Hosp Infect. 2014;86:260-6.

12. Clinical and Laboratory Standards Institute. Performance standards for antimicrobial susceptibility testing: twentieth informational supplement update. CLSI document. M100-MS20. Wayne, PA: CLSI; 2010.

13. European Committee on Antimicrobial Susceptibility Testing guidelines (EUCAST). http://www.eucast.org/clinical_breakpoints/. Accessed 15 May 2015.

14. Meyer E, Schwab F, Schroeren-Boersch B, Gastmeier P. Dramatic increase of third-generation cephalosporin-resistant $E$. coli in German intensive care units: secular trends in antibiotic drug use and bacterial resistance, 2001 to 2008. Crit Care. 2010;14:R113.

15. WHO collaborating centre for drug statistics methodology ATC/DDD index. http://www.whocc.no/atc_ddd_index/. Accessed 26 May 2015.

16. Ministero della Salute. Piano Nazionale della Prevenzione 2014-2018. http://www.quotidianosanita.it/allegati/create_pdf.php?all=9561948.pdf. Accessed 16 April 2015.

17. Ricchizzi E, Morsillo F, Buttazzi R, Pan A, Gagliotti C, Morandi M, et al. Studio di prevalenza europeo su infezioni correlate all'assistenza e uso di antibiotici negli ospedali per acuti. Rapporto nazionale. http://assr.regione.emilia-romagna.it/ it/servizi/pubblicazioni/rapporti-documenti/studio-prevalenza-euro peo-ICA-ospedali-acuti. 2013. Accessed 18 April 2015.

18. Agodi A, Zarrilli R, Barchitta M, Anzaldi A, Di Popolo A, Mattaliano A, et al. Alert-surveillance of ICU-acquired Acinetobacter infections in a Sicilian hospital. Clin Microbiol Infect. 2006;12:241-7.

19. Tan CK, Tang HJ, Lai CC, Chen YY, Chang PC, Liu WL. Correlation between antibiotic consumption and carbapenem-resistant Acinetobacter baumannii causing health care-associated infections at a hospital from 2005 to 2010. J Microbiol Immunol Infect. 2014;pii:S1684-1182(14)00033-4.

20. Meyer E, Jonas D, Schwab F, Rueden H, Gastmeier P, Daschner FD. Design of a surveillance system of antibiotic use and bacterial resistance in German intensive care units (SARI). Infection. 2003;31:208-15.

21. Agodi A, Barchitta M, Cipresso R, Giaquinta L, Romeo MA, Denaro C. Pseudomonas aeruginosa carriage, colonization, and infection in ICU patients. Intensive Care Med. 2007;33:1155-61.

22. Magiorakos AP, Srinivasan A, Carey RB, Carmeli Y, Falagas ME, Giske CG, et al. Multidrug-resistant, extensively drug-resistant and pan drug resistant bacteria: an international expert proposal for interim standard definitions for acquired resistance. Clin Microbiol Infect. 2012;18:268-81.

23. Magiorakos AP, Suetens C, Monnet DL, Gagliotti C, Heuer OE, EARS-Net Coordination Group and EARS-Net participants. The rise of carbapenem resistance in Europe: just the tip of the iceberg? Ant Res Infect Control. 2013;2:6.

24. Ministero della Salute. Circolare "Sorveglianza, e controllo delle infezioni da batter produttori di carbapenemasi (CPE)" del 26.02.2013. http://www.trovanorme. salute.gov.it/norme/renderNormsanPdf;jsessionid=GPGFQ2qMHE8BmEDb7tO5g _..sgc4-prd-sal?anno=0\&codLeg $=45499 \&$ parte $=1 \% 20 \&$ serie $=.2013$. Accessed 26 April 2015.

25. Bush K, Jacoby GA. Updated functional classification of beta-lactamases. Antimicrob Agents Chemother. 2010;54:969-76.

26. European Centre for Disease Prevention and Control. Risk assessment on the spread of carbapenemase-producing Enterobacteriaceae (CPE) through patient transfer between healthcare facilities, with special emphasis on cross-border transfer. Stockholm: ECDC; 2011.

27. Rodríguez-Rojas A, Rodríguez-Beltrán J, Couce A, Blázquez J. Antibiotics and antibiotic resistance: A bitter fight against evolution. Int J Med Microbiol. 2013;303:293-7.

28. World Health Organization. The evolving threat of antimicrobial resistance Options for action. WHO Press, World Health Organization, Geneva, Switzerland, 2012

29. Zou YM, Ma Y, Liu JH, Shi J, Fan T, Shan YY, et al. Trends and correlation of antibacterial usage and bacterial resistance: time series analysis for antibacterial stewardship in a Chinese teaching hospital (2009-2013). Eur J Clin Microbiol Infect Dis. 2014. doi:10.1007/s10096-014-2293-6.

30. Potron A, Poirel L, Nordmann P. Emerging broad-spectrum resistance in Pseudomonas aeruginosa and Acinetobacter baumannii: Mechanisms and epidemiology. Int J Antimicrob Agents. 2015:45:568-85. 